\title{
Rare Case of Intussusception in Young Male who was Using Anabolic
}

\section{Steroids for Muscle Enhancement}

\section{Amandeep Goyal $^{1^{*}} \mid$ Austin Cusisk $^{2}$ | Neil Mast ${ }^{3}$ | Emmanuel A Agaba ${ }^{4}$ | Pankaj Bansal ${ }^{5}$}

\section{*Correspondence: Amandeep Goyal}

Address: ${ }^{1}$ Department of Internal Medicine, Marietta Memorial Hospital, Marietta, Ohio, 45750, USA; ${ }^{2}$ Ohio University Heritage College of Osteopathic Medicine, Ohio University, Athens, Ohio, 45701, USA; ${ }^{3}$ Marietta College Department of Physician Assistant Sciences, Marietta College, Marietta, Ohio, 45750, USA; ${ }^{4}$ Department of Surgery, Marietta Memorial Hospital, Marietta, Ohio,

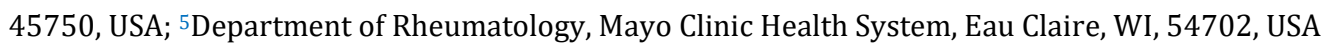

e-mail $\bowtie:$ agoyal@mhsystem.org

Received: 31 March 2020; Accepted: 07 April 2020

Copyright: (C) 2020 Goyal A. This is an open-access article distributed under the terms of the Creative Commons Attribution License, which permits unrestricted use, distribution, and reproduction in any medium, provided that the original work is properly cited.

\section{ABSTRACT}

Intussusception, while a relatively common cause of bowel obstruction in the pediatric population, is relatively rare in adults. In the adult population intussusception is often associated with an underlying tumor or inflammatory disorder of the bowel creating a lead point. We present the case of a healthy 23-year-old male who developed intussusception secondary to anabolic steroid use, tamoxifen, and clomiphene therapy cycles for muscle enhancement. Surgical intervention was utilized, and histopathology of the removed intestine indicated significant mucosal necrosis and omental vascular congestion without evidence of cancer. The patient's anabolic steroid usage likely created a transient polycythemic state. The transient hyperviscous states caused subclinical infarcts yielding lead points for intussusception. Concurrent usage of testosterone and tamoxifen for muscle hypertrophy increases the risk of thromboembolic events.

Keywords: Intussusception, Anabolic steroids, Tamoxifen

\section{Introduction}

Intussusception accounts for only $1 \%-5 \%$ of bowel obstruction seen in the adult population. Whereas idiopathic intussusception is the most common cause of intussusception in children, it is much less common in adults and is often associated with the presence of a lead point (Marinis et al., 2009). Lead points include masses such as diverticulum, adhesions, benign tumors, and malignancy, as well as inflammatory bowel conditions including Celiac disease, Crohn disease, etc. (Marsicovetere et al., 2017). These lesions and bowel inflammation can act as lead points that cause telescoping of the bowel secondary to peristalsis.

Bowel wall ischemia is one cause of bowel wall thickening, which can act as a lead point for intussusception. Ischemic bowel secondary to thromboembolism is one of the many possible side effects 
of anabolic steroids. This occurs as a result of increased hemoglobin concentration that is seen in anabolic steroid users leading to an increased risk of thromboembolic events such as myocardial infarction, stroke, and mesenteric ischemia. It is likely that polycythemic states during active anabolic steroid administration creates subclinical necrosis in bowel acting as the lead points for intussusception development.

Case

A 23-year-old male with no significant past medical history was admitted with worsening abdominal pain and cramping for last 2 days. The patient was noted to have recently completed a 12week cycle of $500 \mathrm{mg}$ Intra-Muscular (IM) testosterone weekly for muscle enhancement after which he began his two-week post cycle therapy of tamoxifen 40mg and clomiphene 50mg daily. Complete blood counts were within upper normal limits with no evidence of metabolic derangements. Computed tomography (CT) of the abdomen showed circumferential thickening beginning at the cecum with colocolonic intussusception of the ascending colon (Fig. 1). No obvious underlying mucosal lesion, obstruction, or masses were evident on CT. Patient underwent urgent exploratory laparotomy, rightsided hemicolectomy, and ileo-transverse anastomosis. The pathology of the specimen was unremarkable for cancerous or inflammatory findings. Pathologic examination of the intussuscepted intestine demonstrated significant mucosal necrosis and omental vascular congestion. Patient did well postoperatively and discharged home with follow-up as needed.

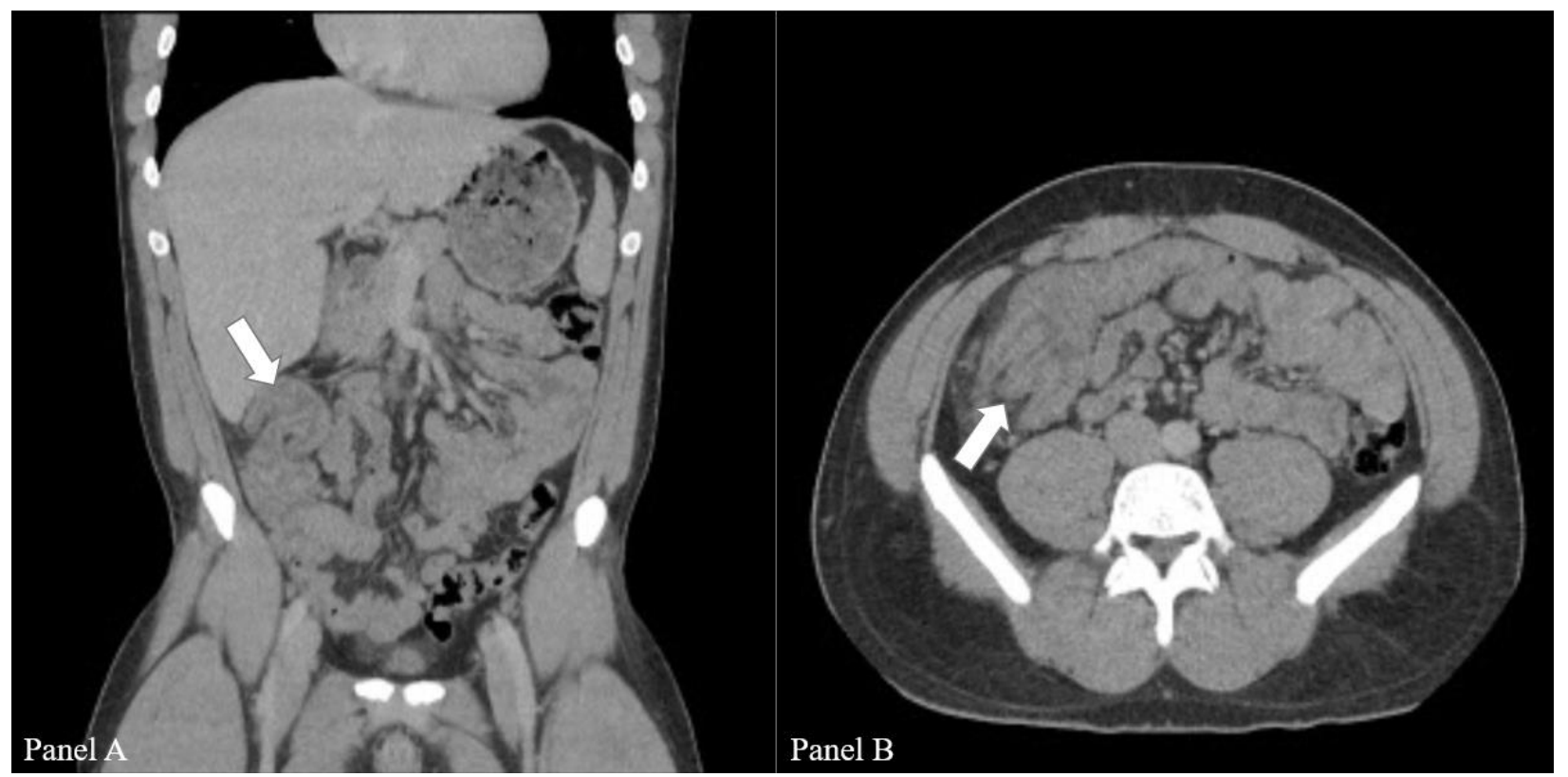

Figure 1: Coronal (Panel A) and Transverse (Panel B) views of the CT abdomen scan demonstrates bowel telescoping as the site of colo-colonic intussusception of the ascending colon (white arrow). 


\section{Discussion}

While there are many psycho-social benefits with muscle enhancement, the adverse effects of androgenic steroid administration may have significant consequences. Well-described effects of exogenous testosterone include polycythemia and erythrocytosis (Vorona and Nieschlag, 2018). The erythrocytosis poses a significantly increased risk of possible thromboembolism with effects including strokes, myocardial infarctions, pulmonary embolism, and deep vein thrombosis (Vorona and Nieschlag, 2018). Furthermore, anabolic steroid use upregulates platelet aggregation by directly stimulating bone marrow. Sustained anabolic steroid usage has many known cardiovascular effects including ventricular hypertrophy, diastolic dysfunction, and arrhythmia. While cardiovascular effects are considerably more dangerous, other adverse effects are described including liver dysfunction, mood irregularity, and reversible spermatogenesis suppression (Vorona and Nieschlag, 2018).

The misuse of other hormonal medications in the pursuit of bodybuilding may have deleterious effects. Estrogen formed from the aromatization of excessive testosterone leads to unwanted feminization, notably gynecomastia. Tamoxifen is used to combat effects of hyperestrogenism by antagonizing selective estrogen receptors (Flynn et al., 2017). Despite tamoxifen's largely antagonistic roles, the therapy retains agonistic properties on other estrogen receptors, contributing to adverse effects (Flynn et al., 2017). Tamoxifen has various described side-effects including thromboembolism. While a rare occurrence, thromboembolic events in male breast cancer patients on 20-40mg daily of tamoxifen are described and often warrant discontinuation of therapy (Wibowo et al., 2016). Clomiphene is also employed in bodybuilding to increase endogenous testosterone production. Clomiphene acting as a Gonadotropin-releasing hormone agonist increases endogenous testosterone without resultant testicular atrophy (Wheeler et al., 2019; Ip et al., 2018). Clomiphene citrate does have a side effect profile including headaches, psychiatric illness exacerbation, and gynecomastia; however, it is not often considered to be thromboembolic.

There is only one case in the literature of a patient abusing anabolic steroids and found to have multiple intussusceptions (Cavanagh et al., 2015). Intussusceptions are very rare in adults and generally associated with mechanical lead points or localized inflammation secondary to a neoplasm or Irritable Bowel Diseases. Our patient had no risk factors besides anabolic steroid cycling with tamoxifen and clomiphene post cycle therapy. Anabolic steroids are well known for increasing the risk of ischemic events including acute MI and bowel obstructions. The known mechanism of ischemic events caused by anabolic steroids is hyperviscosity due to polycythemia, decreased plasma volume, and potentiation of platelet aggregation via direct bone marrow affect (Vorona and Nieschlag, 2018). Additionally, doses of tamoxifen described above would possibly augment the ability to develop thromboembolic events in 
conjunction with testosterone supplementation. In our case, it was possible mesenteric ischemia associated with bowel wall necrosis secondary to transient polycythemic states, potentiation of platelet aggregation due to anabolic steroids, and concurrent effects of tamoxifen therapy that triggered a lead point in developing intussusception.

\section{Conclusion}

Intussusception in adults is often caused by a secondary pathology, like cancer, creating lead points. While a rare entity, anabolic steroids may cause intussusception due to transient polycythemic states causing subclinical thromboembolism in intestinal vasculature. Due to the scarcity of literature, the above described case can serve as a liaison to inform physicians in considering intussusception as a possible differential diagnosis in anabolic steroid abuse.

\section{Author Contributions}

AG: Was crucial in patient healthcare, manuscript construction, co-ordination of publication and literature review.

AC: Was a part of manuscript construction, literature review, publication formatting, and preparation for submission

NM: Played a large role in manuscript construction and literature review

EA: Crucial player in patient healthcare management and co-ordination of manuscript

PB: Helped with final formatting and finalizing draft for proper formatting as per journal specifications

\section{References}

Cavanagh Y, Shah N, Thomas A, Gupta A. Multiple intussusceptions associated with polycythemia in an anabolic steroid abuser, a case report and literature review. Annals of Medical and Health Sciences Research 2015; 5: 368.

Flynn M, Heale KA, Alisaraie L. Mechanism of Off-Target Interactions and Toxicity of Tamoxifen and Its Metabolites. Chem Res Toxicol 2017; 30: 1492-507.

Ip EJ, Doroudgar S, Lau B, Barnett MJ. Anabolic steroid users' misuse of non-traditional prescription drugs. Research in Social and Administrative Pharmacy 2019; 15: 949-952.

Marinis A, Yiallourou A, Samanides L, Dafnios N, Anastasopoulos G, Vassiliou I, et al. Intussusception of the bowel in adults: A review. World Journal of Gastroenterology 2009; 15: 407.

Marsicovetere P, Ivatury S, White B, Holubar S. Intestinal Intussusception: Etiology, Diagnosis, and Treatment. Clinics in Colon and Rectal Surgery 2016; 30:030-9.

Vorona E and Nieschlag E. Adverse effects of doping with anabolic androgenic steroids in competitive athletics, recreational sports and bodybuilding. Minerva Endocrinol 2018; 43:476-488. 
Wheeler KM, Sharma D, Kavoussi PK, Smith RP, Costabile R. Clomiphene Citrate for the Treatment of Hypogonadism. Sexual Medicine Reviews 2019; 7: 272-276.

Wibowo E, Pollock PA, Hollis N, Wassersug RJ. Tamoxifen in men: a review of adverse events. Andrology 2016;4: 776-788. 